\title{
Adaptive Passive Control of Dynamic Response Through Structural Loading
}

\author{
Jonathan L. du Bois*, Nick A. J. Lieven ${ }^{\dagger}$ and Sondipon Adhikari ${ }^{\ddagger}$ \\ University of Bristol, Queens Building, University Walk, Bristol, BS8 1TR, England
}

This paper addresses the issue of the effect of structural load on structural dynamic behavior, and how an understanding of this behaviour can be used beneficially to reduce the amplitude of response. The processes discussed lead to a new method of adaptively retuning structures through the introduction of known loads, with particular reference to helicopter response suppression.

In many applications it is desirable to reduce the response of a structure without the use of damping. This has been the subject of much research with regard to helicopter design. Passive methods exist but do not allow for multiple excitation frequencies, shifting frequencies or structural alterations arising from different flight configurations and loading. Active systems have the advantage that they can respond quickly and continuously to changes in system configuration but they require significant power and higher levels of maintenance than passive systems. Adaptive passive or semi-active systems are able to adapt to flight conditions while using far less power than fully active systems. They generally involve similar techniques to passive methods with some mechanism for tuning the parameters of the vibration absorber. In this paper, numerical and experimental techniques are used to investigate the effect of loading on structural response with a view to implementing a structural load based adaptive passive device.

To set the scene for this proposal, a case study of structural loading on a helicopter tailboom is presented, illustrating the extent to which the dynamic response can be affected by loading. Finite element techniques are investigated and the results compared with experimental data. The difficulties in modelling the changes in dynamic performance are discussed with refence to a case study on joint parameterization. It is found that parameters updated for one load case may not apply across an entire loading regime.

A preliminary study is then carried out to assess the feasibility of the proposal, and it is demonstrated that such a system can provide improved response with low power requirements across a range of narrow band excitation frequencies. This important result provides a starting point for further research into automated adaptive techniques for response suppression, and critical areas for new work to be focused on are identified.

\section{Nomenclature}

$K_{T} \quad$ tangent stiffness matrix

$K_{E} \quad$ elastic stiffness matrix

$K_{G}$ geometric stiffness matrix

$u, v$ axial and transverse beam displacements

$E \quad$ Young's modulus

A cross-sectional area

$L \quad$ length

$\varepsilon_{x}, \varepsilon_{y}$ axial and transverse elemental strain

*PhD Student, Department of Aerospace Engineering, University of Bristol, Student Member Grade.

${ }^{\dagger}$ Head of Department, Department of Aerospace Engineering, University of Bristol

${ }^{\ddagger}$ Lecturer, Department of Aerospace Engineering, University of Bristol 


\section{Introduction}

Adaptive passive response suppression systems provide an alternative to damping which is a compromise between power-intensive active systems and low-performance passive systems. They generally work on the same principles as passive devices such as tuned mass dampers, where the response of the structure is tuned to a desirable state by the correct adjustment of the parameters of the device. The difference lies in the ability of the adaptive device to adjust these parameters whilst in service, in response to changing excitation or environmental factors. In this paper, numerical and experimental techniques are used to investigate the effect of loading on structural response with a view to implementing a structural load based adaptive passive device.

Structural loading induces an effect known as stress stiffening. This is a manifestation of geometric nonlinearity, whereby the transverse stiffness of a beam or shell is affected by applied axial or membrane loads. This has been used for millennia in stringed musical instruments, where the pitch is varied by tensioning the strings. It is also illustrated by the well-known Euler buckling instability, a special case of the generalised result provided by, for example, Warburton ${ }^{1, \mathrm{p} .183}$. Slender structures are designed primarily to carry only axial loading. As such, the effect of stress stiffening on static displacement is usually minimal. The dynamic response of the structure, however, can be greatly influenced by the change in transverse stiffness. This was exploited as far back as 1936, when Stephens ${ }^{2}$ explained how the natural frequencies of a structure can be used to determine the load carried in its members. Internal pre-stressing of a framed structure will cause some members to experience compression while others are placed in tension. The contrasting effects on stiffness of the members creates a structure with both rising and falling natural frequencies.

As mentioned, in many applications it is desirable to reduce or alter the response of a structure without the use of damping. This can be to avoid the undesirable side effects of damping, such as increased response away from resonance, or for situations where damping alone is impractical or insufficient. This has been the subject of much research with regard to helicopter design. Tuned vibration absorbers (TVA), ${ }^{3}$ sometimes referred to as undamped vibration absorbers (UDVA) or dynamic vibration absorbers (DVA), are a passive solution to this problem; in the simplest case these consist of a mass and spring attached at a problematic part of the structure with the spring stiffness tuned to absorb the vibration energy at the frequency in question. This works well in situations where the excitation frequency is fixed and known a priori (for example a fixed-speed helicopter rotor) but does not allow for multiple excitation frequencies or shifting frequencies. It also requires the rest of the structure to remain relatively unchanged and does not allow easily for alterations inevitably arising from different flight configurations and loading.

Another approach is to use an active system such as higher harmonic control (HHC), active isolation or active control of structural response (ACSR). ${ }^{4}$ The latter two use the idea of cancelling vibration-induced structural forces with actuators in the structure. In active isolation, they are used in the load path between two parts of a structure, thus isolating one part from a source of excitation in the other (for example, isolating the fuselage from the rotor assembly). In ACSR the actuators are placed at strategic points in the structure and the vibrations they create are superimposed on those created by the rotor to affect the response as a whole. HHC is employed immediately at the site of excitation: the blade pitch is varied at higher harmonics of the passing frequency to directly reduce excitation at the source. These systems all have the advantage that they can respond quickly to changes in system configuration. Their behaviour is determined by feedback loops incorporating sensors on the structure, thus continuously adapting to the required input. The disadvantages are that they require continuous power and higher levels of maintenance than passive systems. In the event of power interruption or component failure they will cease to have an effect on the dynamic performance.

The middle ground is to use adaptive passive or semi-active systems. These employ the same method of response control as a TVA but incorporate a method of adjusting the parameters (usually the stiffness) of the absorber and thus the frequency of effective operation. State-switched absorbers (SSA) ${ }^{5}$ and switch-shunted dampers (SSD) contain piezo-ceramic elements whose stiffness is determined by the open or closed state of the connecting circuitry. By switching between states the operational bandwidth of the device is increased. Other adaptive passive systems use electromagnetic elements to adjust the stiffness of a TVA continuously, ${ }^{6}$ providing for operation over a range of system configurations. These devices require power to change state but can operate passively in one state with no power supply.

In the text that follows, the argument for using preload to deliberately affect the dynamic response is developed with the aid of experimental data. Consideration is given to analytical modelling techniques to determine optimum implementation of the preloading, and these are demonstrated with more practical 
examples. An autonomous controller is then implemented to perform the task of response suppression using quasi-static loads applied by an actuator embedded in the test structure. The difficulties it presents are discussed and suggestions as to how to approach these in future work are proposed.

\section{Lynx Tail Boom Variability Studies}

As part of a collaborative project to improve the Finite Element (FE) modelling of a WHL Lynx Mk 7 helicopter, a variability study of the structure was carried out. ${ }^{7}$ Some of the findings are summarised here. Three key factors were identified as contributing to the variability of the results:

- variability in the modal extraction techniques

- variability in the manufacturing of nominally identical parts

- variability in environmental factors

The final consideration, environmental factors, encompasses temperature and humidity changes but perhaps the most significant environmental factor affecting structural response is service loading. The tail boom on the Lynx, or indeed any rotorcraft with a single main rotor and tail rotor configuration, carries a significant load to oppose the torque produced by the main rotor. If the steady lateral force generated by the tail rotor is considered in isolation, the load carried in the tail boom can range from $2.4 \mathrm{kN}$ to $7.1 \mathrm{kN}$. With this in mind, tests were devised to determine the extent of the effect of lateral preloading on the dynamic behaviour of the boom.

The setup can be seen in figure 1. The tail boom is separated from the main body of the fuselage to simplify the test process and to isolate the source of contributions to changes in behaviour. It is mounted rigidly on a reaction wall at its root, and a tether extends laterally from the tailrotor attachment point to a rigid stay. Connected in line with the tether are a load cell and a turnbuckle. Tightening the turnbuckle creates tension in the tether, resulting in a lateral load on the tail boom.

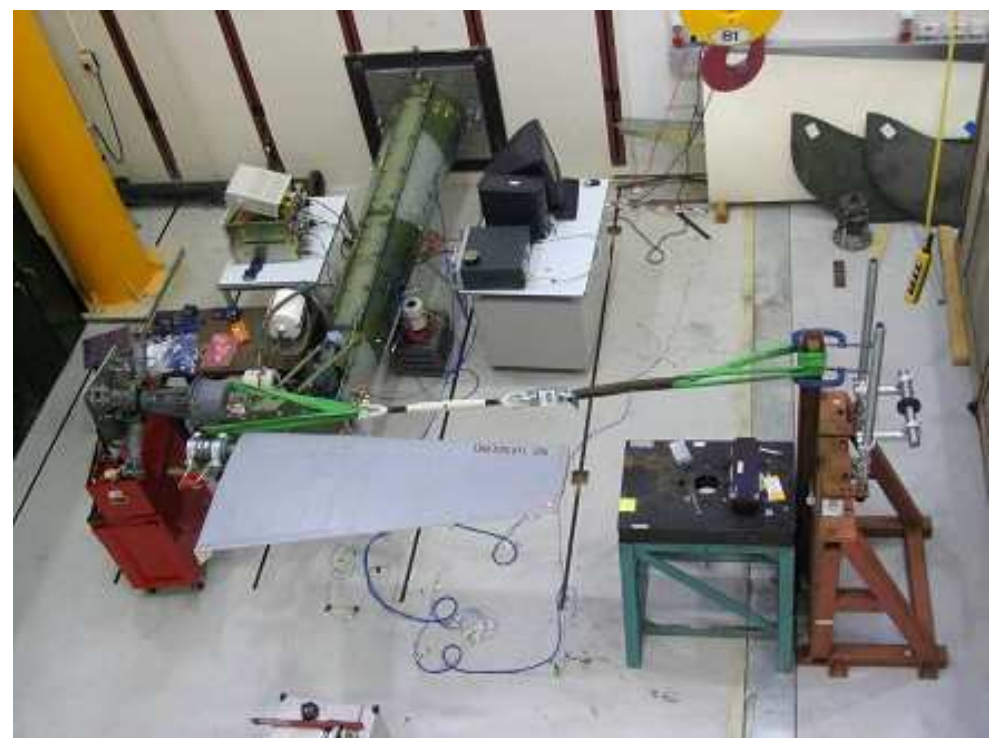

Figure 1. The lateral turnbuckle loading test setup

The dynamic excitation was provided by two shakers: one mounted at the intermediate gearbox in the lateral boom axis (in local body coordinates), and the other attached to a skin panel approximately half way along the boom at the site if an internal stiffening rib. Force transducers were mounted between the shakers and the boom, and accelerometers were distributed around the structure at locations selected manually from observations of previous test data. Burst random noise was used to obtain the frequency response functions (FRFs) in the range 0 to $256 \mathrm{~Hz}$ with linear averaging over ten runs.

Lateral point accelerances for the boom under forces ranging from $50 \mathrm{kgf}$ to $600 \mathrm{kgf}$ can be seen in figure 2 . The results from this test show that the tail boom response exhibits substantial variation under load. 


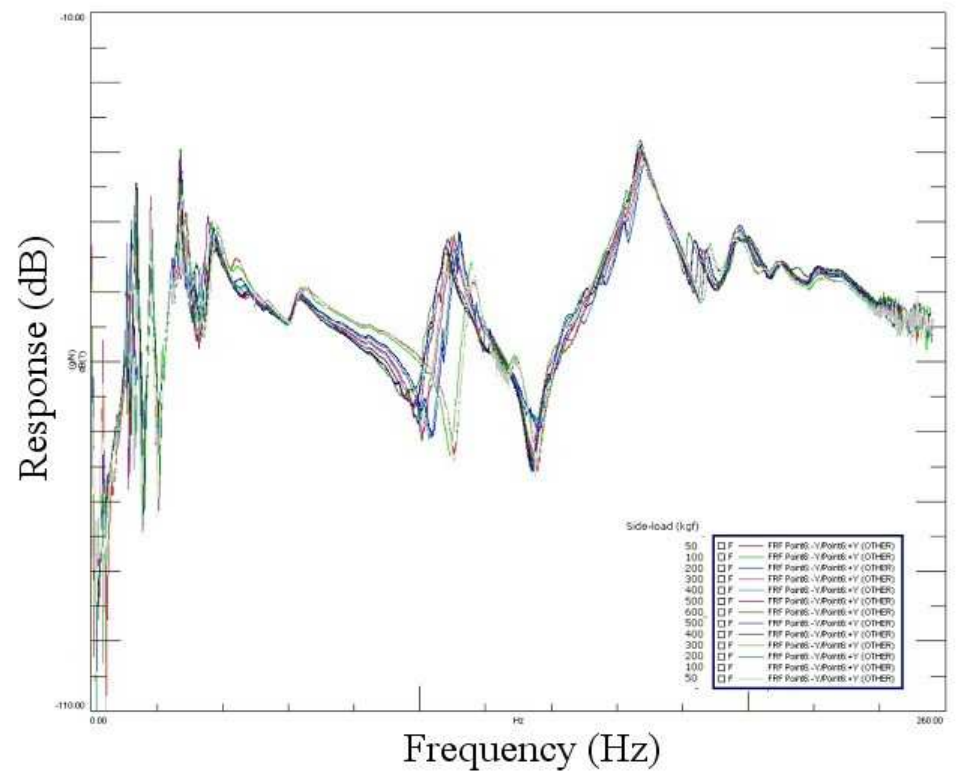

Figure 2. Plotting the point FRFs for lateral loading cases between 50kgf and 600kgf shows considerable variation in response with loading.

The consequences of these findings are twofold. Firstly, operational stresses on a structure will have a significant effect on the dynamic characteristics of the structure. This is particularly true for aircraft, where the loads imposed are high relative to the stiffness of the structures, and even more relevant to rotorcraft, whose rotors produce high oscillatory forces which must not coincide with the resonant frequencies of the fuselage. In light of this, a need is identified for detailed operational load estimation to better understand the dynamics of rotorcraft in flight.

The second ramification is that although incidental loading may cause undesirable and unpredictable modifications to the FRFs, the delierate introduction of known loads to a structure could produce beneficial alterations to the dynamic response. This idea is explored in the following sections.

\section{Stress Stiffening: Numerical and Experimental Verification}

The changes witnessed in the dynamic response of a structure under loading is caused by nonlinearity. In general, structures will display an almost linear variation of displacments over the majority, if not the entirety, of their loading regime. Large forces, slender structures and certain joint configurations will, however, lead to nonlinear load-displacement behaviours, classified as geometric nonlinearities. ${ }^{8}$ Nonlinearities are characterised on a load-displacement graph by the changing gradient of the curve. The stiffness at a given point is represented by the tangent to the curve and referred to as the tangent stiffness. ${ }^{9}$ Geometric nonlinearities are commonly included in static structural problems, and are generally split into two categories: stress stiffening and large deformation. In finite element analyses, the latter is usually subcategorised further into large elemental rotations and large elemental strains. In dynamic analyses, stress stiffening is by far the most important factor for lower loads and small deformations, where its effect on dynamic response is belied by its relative insignificance in static solutions. As structural deformation increases it also becomes necessary to take elemental rotations into account, but the further complication of large elemental strain is easily avoided in simple models through refinement of the spatial discretisation. A summary of the different formulations useful in dynamic analyses is given by Mayo et al. ${ }^{10}$

The problem being considered here is that of the change in the linear dynamic response under nonlinear static loading. The approach adopted is to first use a nonlinear static analysis to determine the tangent stiffness for a given load case. Then, assuming small dynamic loads and displacements, the tangent stiffness is used to obtain a linear dynamic solution for this load case. 


\section{III.A. Experimental Arrangement}

In order to correlate experimental data with FE theory a simple construction is chosen to demonstrate the principles. A redundant structure was designed in the form of a cross-braced rectangle, pictured in figure 3. The corner joints are welded, as this connectivity introduces the least uncertainty to the structure. Two bolts in one of the diagonal members pass either side of the other cross-beam so there is no contact between the two members. When the bolts are tightened, they shorten that member, resulting in a load transmitted throughout the structure, with the diagonal members in tension and the outside members in compression. To simplify the FE analysis the beams are arranged to behave as a 2-dimensional system. The second moment of area of each beam in the plane of the structure is far smaller than that perpendicular to the plane so the vibration modes out of plane will occur at much higher frequencies than those in plane. All of the beam centrelines lie in one plane to avoid coupling between in-plane and out-of-plane modes.

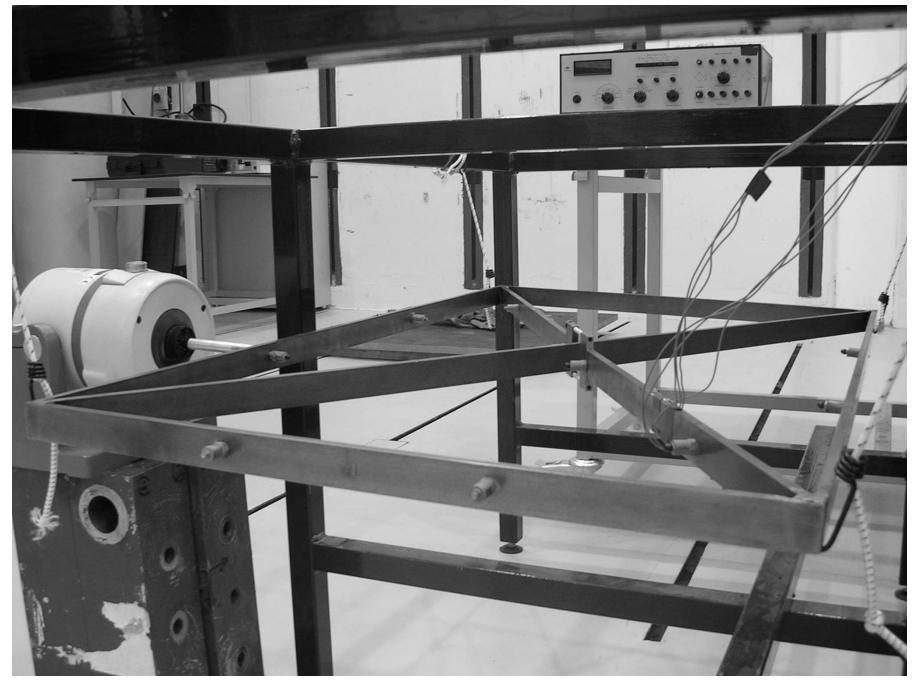

Figure 3. The redundant frame with tightening mechanism in one of the diagonal members. The two diagonals are not connected where they cross. The corner joints are rigidly welded.

The framework is suspended by elastic bungees to approximate free vibration conditions and fourteen accelerometers are attached. In order to control the loading of the structure, strain gauges are mounted on top and bottom of the adjustable member. By balancing the two gauges it is possible to ensure that no bending moment is induced in the structure by uneven tightening of the two tensioning bolts. A single shaker is used to supply broadband excitation to the structure and obtain the FRFs and modal response. The modal characteristics are identified using a least squares complex exponential parameter estimation technique in the time domain with multiple degrees of freedom.

\section{III.B. Finite Element Model}

The FE model uses 6 two-dimensional Euler-Bernoulli beam elements ${ }^{11(p .81)}$ to represent each member. The tightening mechanism is represented by a further 3 elements. The static loading is modelled by removing the coupling between the bolts and the frame at one end of the mechanism. The free end of the frame is fully constrained and the free end of the bolts is constrained in the transverse and rotational degrees of freedom (DOFs), leaving the axial DOF unconstrained. A load is then applied axially to this end. Once the static solution is obtained, the two free ends are rigidly joined again and the constraints removed to obtain the free dynamic response using the new tangent stiffness.

It is common to derive a geometric stiffness matrix based on axial load which, when added to the normal elastic stiffness matrix $K_{E}$, gives the tangent stiffness:

$$
K_{T}=K_{E}+K_{G}
$$

Jennings ${ }^{12}$ uses a series of transformations to include both stress stiffening and large deformation effects in the stiffness matrix. He derives an exact solution and a computationally efficient approximation based on 
second order expansions of the axial strain term. Using his second order approximation a geometric stiffness matrix can be formed in terms of the axial and transverse end displacements:

$$
K_{G}=\frac{E A}{L}\left[\begin{array}{cccccc}
0 & \varepsilon_{y} & 0 & 0 & -\varepsilon_{y} & 0 \\
\varepsilon_{y} & \left(\varepsilon_{x}+\frac{3}{2} \varepsilon_{y}^{2}\right) & 0 & -\varepsilon_{y} & -\left(\varepsilon_{x}+\frac{3}{2} \varepsilon_{y}^{2}\right) & 0 \\
0 & 0 & 0 & 0 & 0 & 0 \\
0 & -\varepsilon_{y} & 0 & 0 & \varepsilon_{y} & 0 \\
-\varepsilon_{y} & -\left(\varepsilon_{x}+\frac{3}{2} \varepsilon_{y}^{2}\right) & 0 & \varepsilon_{y} & \left(\varepsilon_{x}+\frac{3}{2} \varepsilon_{y}^{2}\right) & 0 \\
0 & 0 & 0 & 0 & 0 & 0
\end{array}\right] \quad \text { where } \begin{aligned}
& \varepsilon_{x}=\frac{u_{2}-u_{1}}{L} \\
& \varepsilon_{y}=\frac{v_{2}-v_{1}}{L}
\end{aligned}
$$

and $u$ and $v$ are the axial and transverse displacements, $E$ is the Youngs Modulus, $A$ the cross-sectional area and $L$ the length of the beam.

Closed form solutions for general nonlinear structural problems are rare and iterative techniques are usually employed. Commonly in dynamic computations, the tangent stiffness is determined by running a single linear static analysis and employing the load distribution obtained from this to compute the stress stiffening matrix. While this is often adequate for small loads, there are notable exceptions where the geometry changes significantly through structural deformation, or where the new stiffness matrix causes significant changes in stress distribution. A more accurate method, employed here, is to calculate the displacement at small load intervals using linear theory, evaluating a new tangent stiffness at each step. Newton-Raphson iterations ${ }^{13(\mathrm{p} .277)}$ are employed to reduce the residual force at each load increment.

\section{III.C. Results}

The structure was tensioned, tightening the bolts incrementally until the point was reached where further tightening produced little increase in the strain measurements and the structure was deemed to have buckled. The experimental eigenfrequency loci can be seen in figure 4 .

Comparing these curves with the results from the initial FE model in figure 5(a), the two data sets clearly follow the same trends for low loads but differences emerge as the buckling load is approached. It was found that the eigenfrequiency loci near buckling are very sensitive to the initial configuration. Figure 5(b) shows the results for the same FE model run with an initial transverse curvature in the long outside members in the form of a half sine wave. This perturbation allows the FE model to behave more like the real structure with its inevitable imperfections. The initial displacement of the beam centres is just $0.185 \%$ of the member length but produces a marked change in the dynamic

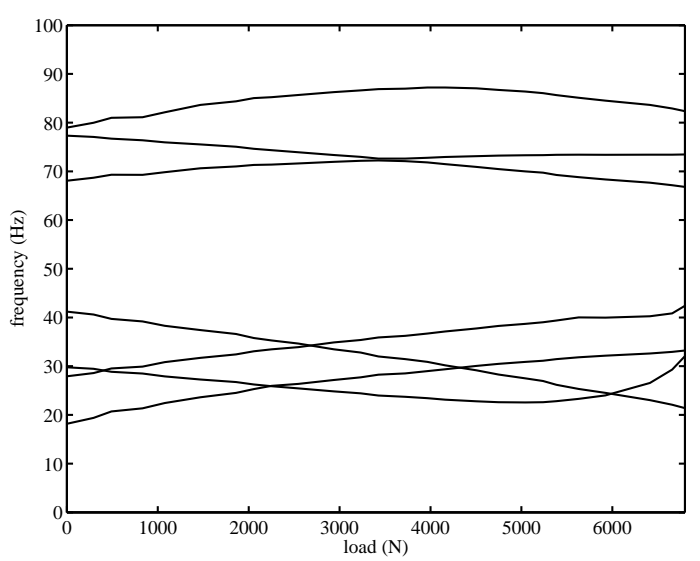

Figure 4. Experimental eigenfrequency loci plotted with varying load. behaviour close to buckling. Whereas the ideal structure deforms linearly before suddenly failing, the onset of buckling in the perturbed structure begins earlier and develops more gradually. The implication of this in terms of modelling the dynamic behaviour of structures near the buckling load is that the deflection of the structure plays a critical role in these regions and single-step stress stiffening calculations, as mentioned in the preceding section, will not produce accurate results.

The motivation behind this work is to correctly model and then reduce the response of a structure. Accordingly, in figure 6 the frequency response of the FE model is compared to that of the test structure over the loading range. The similarity of the two graphs is striking and the key features of the experimental data are easily picked out on the simulated results. This serves as a demonstration of the validity of the stress stiffening techniques used in the FE model. There are several interesting artefacts in these plots. Most notable is the large dip in response where the two prominent modees meet near the center of the graph. Although intereesting, this result is both unusual and confined to a specific point on the strucutre, and is not likely to provide any useful response suppression techniques in general structures. More information on the causes of this effect is given by Igusa. ${ }^{14}$ Some interesting analysis of the behaviour in this region is given by du Bois et al. ${ }^{15}$ 


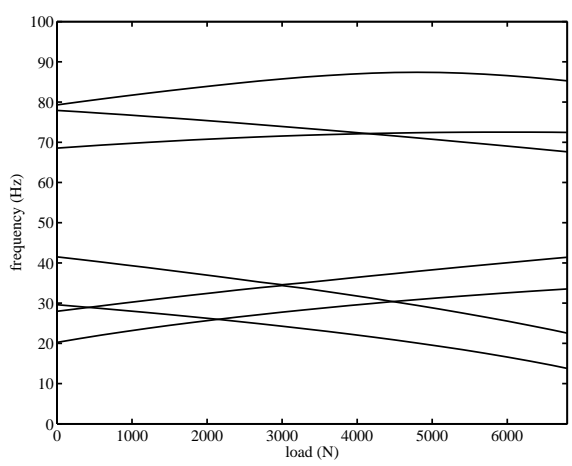

(a) Initial FE model

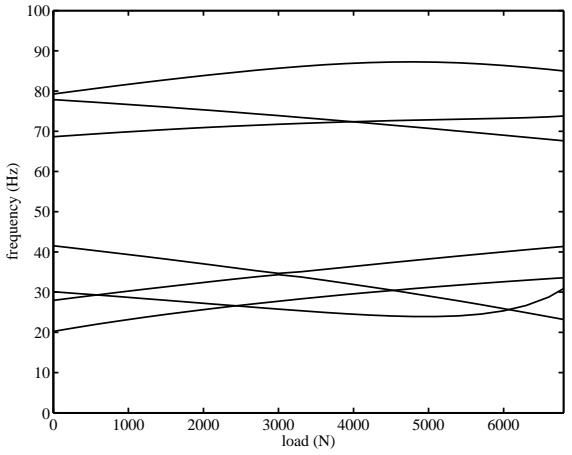

(b) Perturbed FE model

Figure 5. Eigenfrequency loci plotted from finite element data at $300 \mathrm{~N}$ load intervals. A small initial perturbation of the side members results in significantly different behaviour close to the buckling load.

Two further possibilities present themselves for reducing the response:

- shifting the natural frequencies outside the range of the excitation frequencies

- aligning an antiresonance with the excitation frequency at key structural locations

Using antiresonances to control response at specific regions of the structure is entirely feasible, but can only have limited benefit as antiresonances vary from point to point in a given structure. The possibility of moving the resonant frequencies to avoid coincidence with the excitation frequencies, however, is far more generally applicable and would have beneficial effects throughout the structure. This is the technique that is investigated further in the sections that follow.

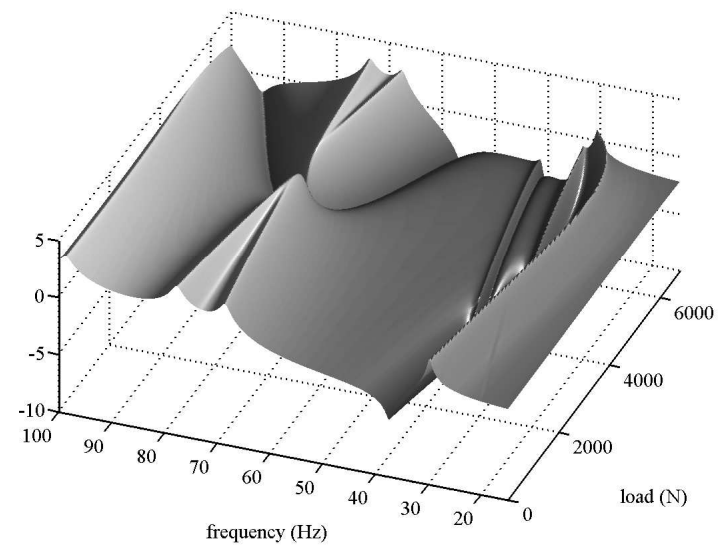

(a) Finite element model data

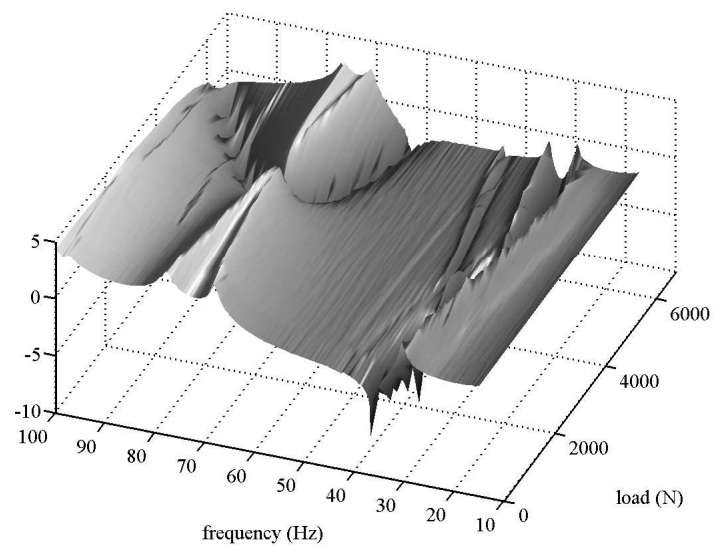

(b) Experimental data

Figure 6. Waterfall plots for the cross-braced rectangle showing the variation of the FRFs with load

\section{III.D. Dynamic Behavior of Joints Under Loading}

There is one more important demonstration offered by this simple structure. Structural joints continue to be responsible for the majority of the uncertainty and inaccuracies in dynamic FE models. Preloaded structures are no exception, and with this in mind a second structure was tested, differing from the first only by the inclusion of pinned joints. In place of the welded joints at the corners, interleaved hinges were incorporated as pictured in figure 7. These were initially modelled as pin joints in the FE model, but it was found necessary to update small torsional stiffness parameters at the joints to account for sticktion. These represented the sticktion at the three interfaces at each of the corner joints: between the two outside members; between 
the long outside member and the diagonal; and between the short outside member and the diagonal. A weighted penalty function method ${ }^{16}$ was used to create an updated model with very good correlation for both resonant frequencies and mode shapes (where the mode shape correlation was ascertained using the modal assurance criterion ${ }^{17}$ ). These results are shown in figure 8.

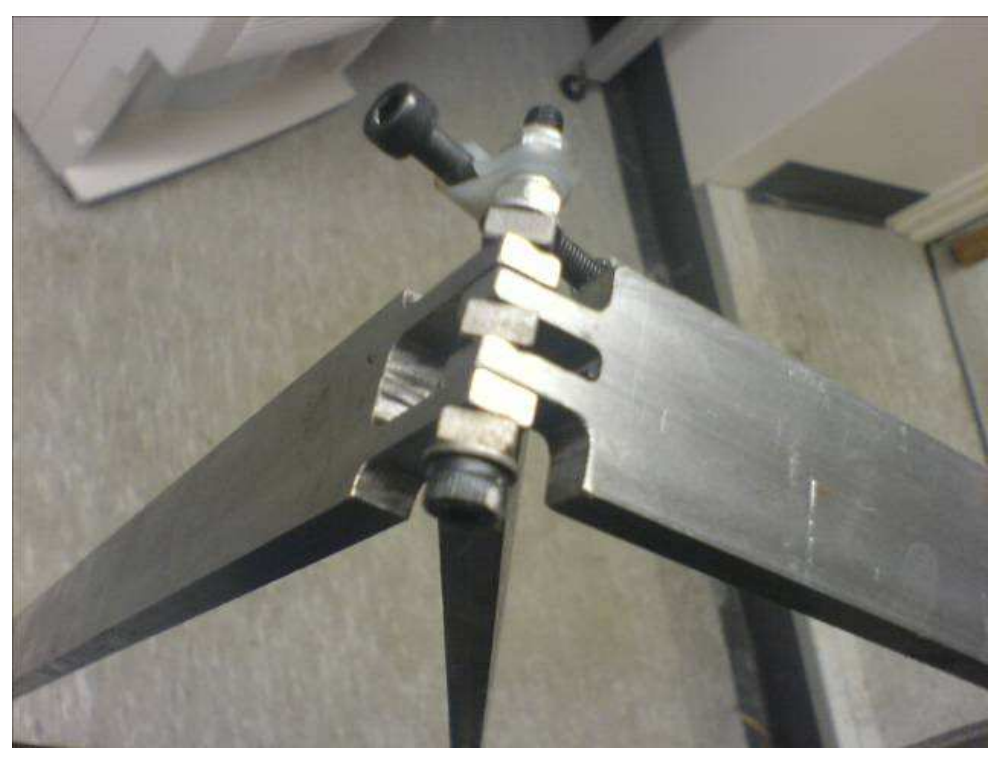

Figure 7. The three-way interleaved pin-joint at the corner of the cross-braced rectangle
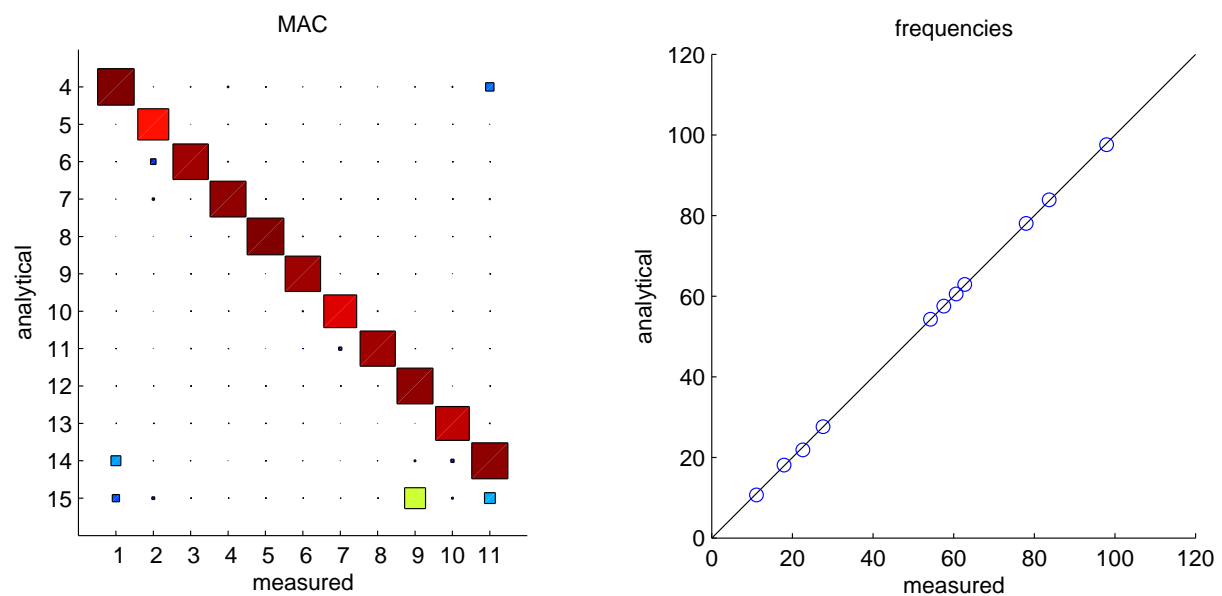

Figure 8. Modal Assurance Criteria and Correlated Mode Pair Comparisons between measured and analytical modes in the pin jointed structure for the zero load case.

The experimental frequency data from this structure is compared with the initial FE results throughout the loading range in figure 9. The same trends are apparent in each graph but there is a marked difference in the gradient of the two data sets. It was thought that this could be due to an increase in sticktion as the loading on the joints was increased. To investigate this further, the torsional stiffness parameters at the joints were updated separately for each load step. Their updated values and the corresponding frequency loci are plotted in figure 10(a). It is posited that linear trends in the parameters exist, masked by the erratic slipping of the joints as they are loaded. To test this, a final model was updated just once based on all available loadsets, this time with six universal parameters defining three linearly load-dependent torsional springs at the joints. The results are shown in figure 10(b), and correlate very well with the experimental data. This investigation highlights the need for careful consideration of the influence of loading on joint parameters. 

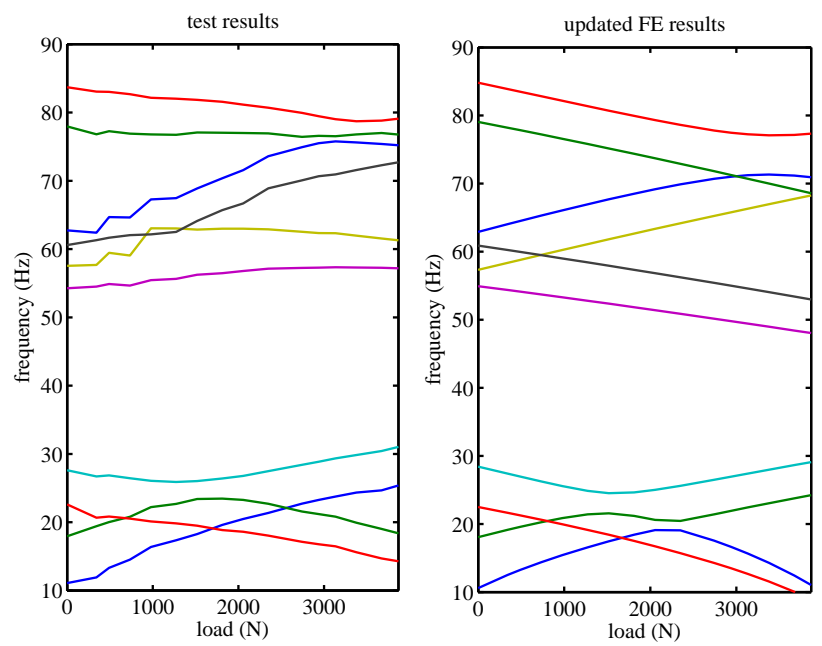

Figure 9. Comparison of frequency loci over the loading spectrum obtained from experimental data and from the FE model
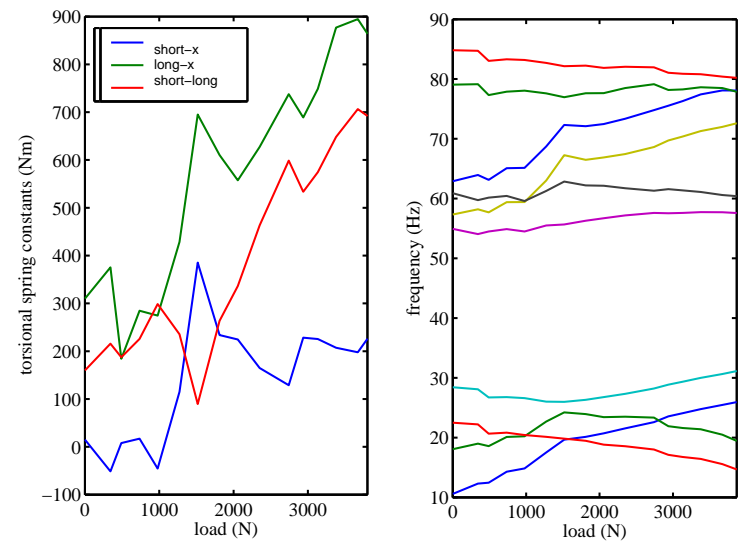

(a) Joint parameters updated at each load step
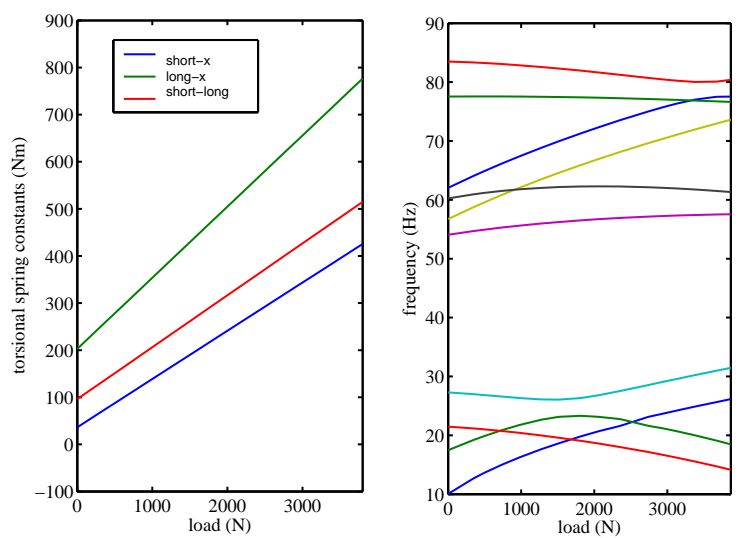

(b) Overall parameters updated to give linear variation of joint parameters with load

Figure 10. Torsional stiffness parameters at joints after updating, along with the corresponding frequency loci.

\section{Automated Control}

\section{IV.A. Proposal}

In this section a preliminary study into the feasibility of an automated load-based adaptive-passive device is made. It is proposed that the three key components of such a system are:

- An actuator capable of maintaining a load defined by the input signal

- A real-time estimation of the excitation frequency

- A decision-making or route-planning algorithm to determine the optimum actuator load for a given excitation frequency

\section{IV.B. Implementation}

The structure chosen to implement the device is an adaptation of the Meroform ${ }^{\circledR}$ space frame structure used by Mottershead et al. in assessing various updating techniques. ${ }^{18}$ The advantages of this structure are 
that it is already relatively well understood and is easily adapted or extendend, being comprised of modular components.

The structure is pictured in figure 11. One of the diagonal members on the top of the frame has a linear ball screw actuator and a load cell incorporated into it. The section of the structure where it is located is deliberately designed to be statically determinate without that member. Once included, there is a redundancy which allows the internal prestressing to be achieved. Without this redundancy the structure would deform under load. Although the dynamic performance would be altered in this case, it would be primarily due to geometric reconfiguration and the stress stiffening achieved would be negligible. It is desirable to retain a relatively undeformed structure, hence the redundancy is necessary.

The actuator is powered by a DC motor. The load cell consists of two strain gauges either side of a waisted steel bar. These are employed in opposite sides of a Wheatstone bridge ${ }^{19}$ and the bridge voltage is routed through amplifying and signal conditioning hardware to measure the axial tension in the member, accounting for any bending. Using a rapid controller prototyping software suite in conjuction with analogue-to-digital and digital-to-analogue converters (ADCs and DACs), a closed loop control system is created incorporating the actuator and the load cell. With this setup the actuator load can be directly assigned any value between 0 and $6 \mathrm{kN}$.

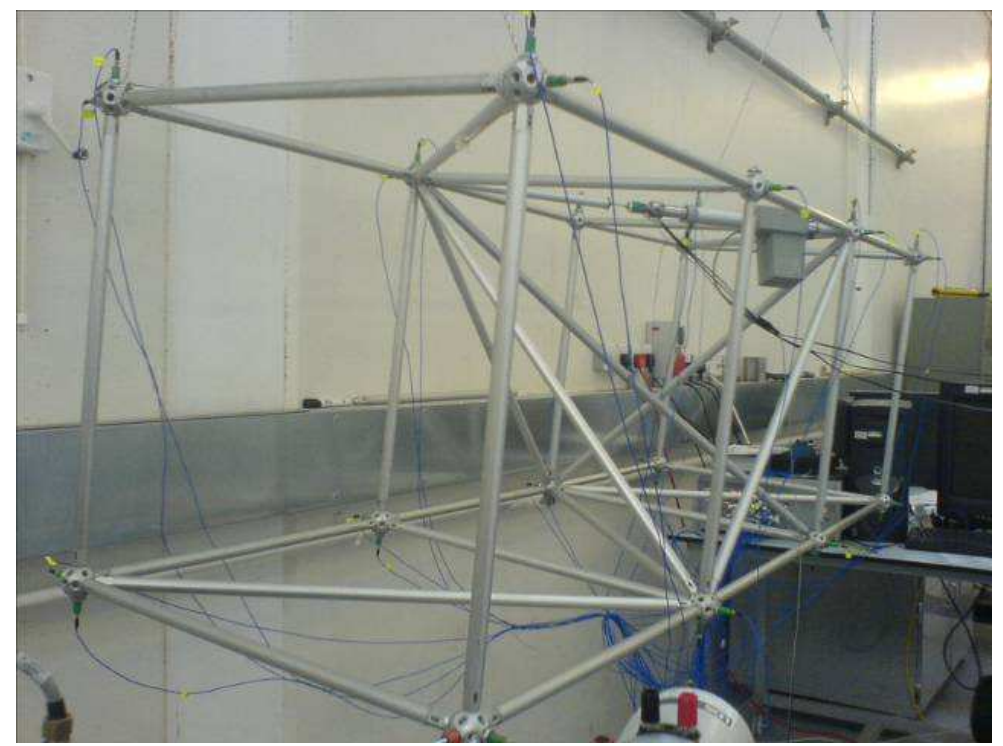

Figure 11. The test structure for the automated adaptive response suppression system. The actuator can be seen mounted at the top of the framework, which is supported on bungees from above to approximate free vibration conditions.

Thirty-one accelerometers are distributed around the structure to measure the frequency response. A thirty-second accelerometer is connected to the rapid prototyper for the purpose of real-time estimation of excitation frequency. This is the only sensor used by the control system; the rest are purley for measurement purposes. The frequency estimation is achieved fairly crudely by applying a short-time, fast Fourier transform (ST-FFT) in conjunction with a Hamming window to short bursts of data sampled from the accelerometer. ${ }^{20}$ A power spectral density (PSD) is calculated and the peak frequency is taken to be the primary excitation frequency. The method was chosen for its simplicity, and provides reasonable accuracy where one clear peak exists but exhibits poor ability to choose the most appropriate value where multiple peaks or a broad spectrum of response exist.

A hammer test is performed to gather response data averaged over three runs for each load, in steps of $150 \mathrm{~N}$ from 0 to $2250 \mathrm{~N}$. Figure 12 shows the FRFs for a response point located on the cubic diagonal member in the framework. A key feature of this plot is the clearly dominant mode for this point at around $50 \mathrm{~Hz}$. As the applied load increases, the frequency of this mode decreases. This is an example of exactly the behaviour it is hoped to exploit. Many of the other modes change frequencies also. The approach taken to the decision making process for this preliminary study is simple: a path is determined that avoids as many of the resonances as possible. At each frequency, a load is identified where the response is lowest. This is illustrated in figure 13. Currently this analysis is performed manually but methods of automation to be 
developed are discussed in section IV.D. The values obtained from this process are incorporated into the control system to create a lookup table capable of returning the the optimum actuator load for any single excitation frequency in the range 0 to $200 \mathrm{~Hz}$.

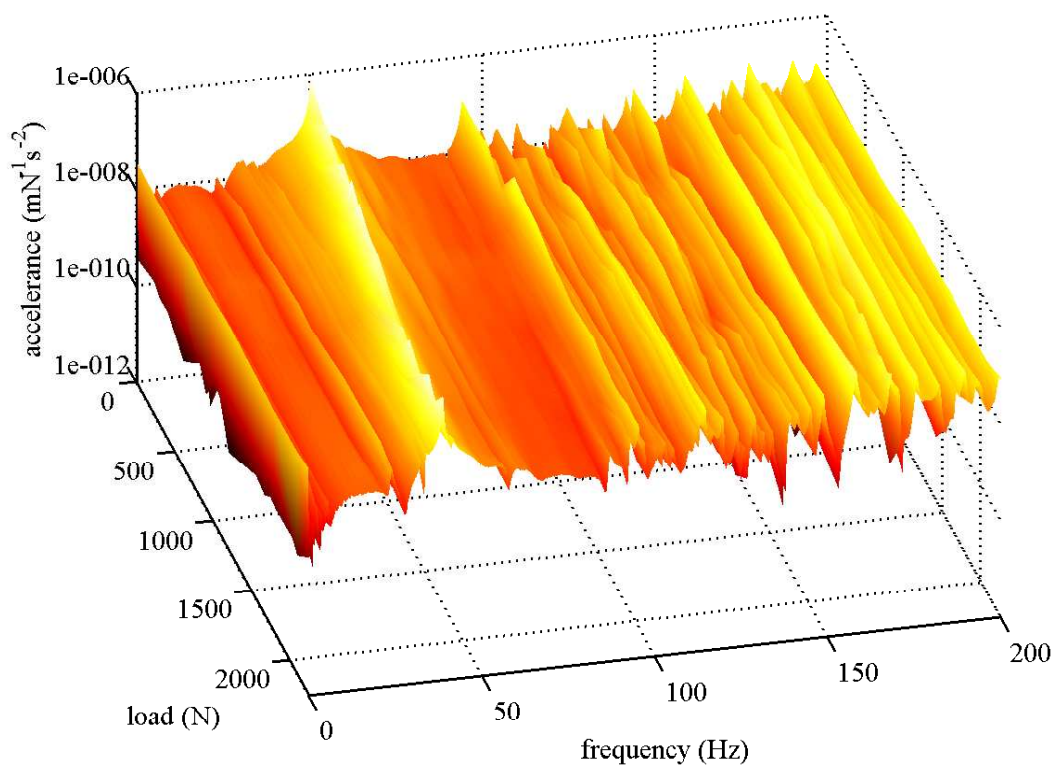

Figure 12. Waterfall plot of an FRF for the Meroform ${ }^{\circledR}$ structure under actuator loads ranging from 0 to $2250 \mathrm{~N}$

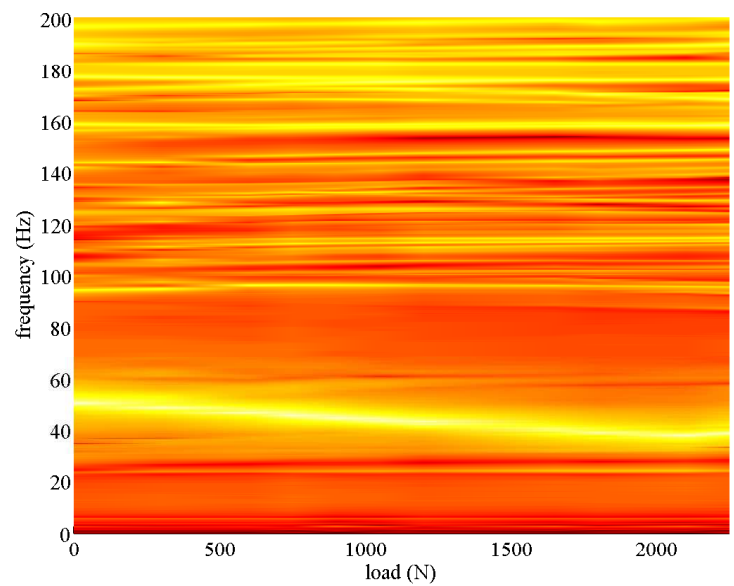

(a) Plain FRF contours

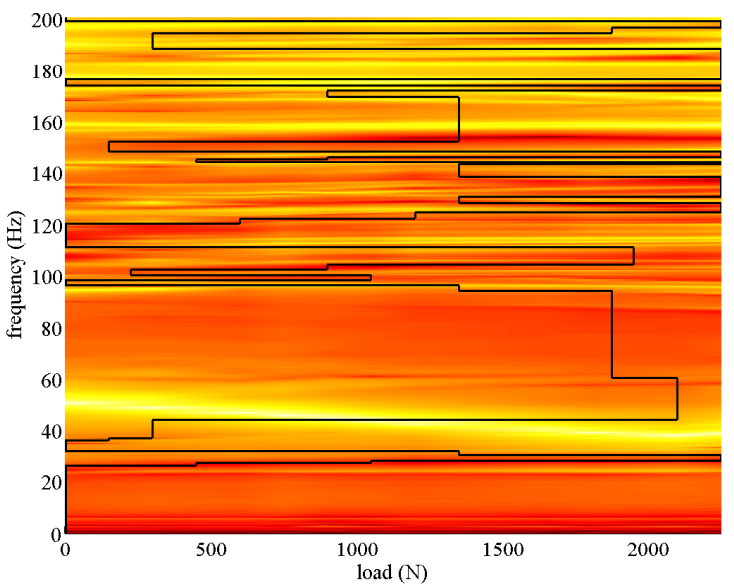

(b) FRF with optimised load path overlaid

Figure 13. Waterfall plots for the Meroform ${ }^{\circledR}$ structure, viewed from above with light regions representing high response and dark regions represening low response

\section{IV.C. Testing}

Having implemented and tested all three components, they are assembled to form the complete self-contained system. It is expected that this system will identify and implement the optimum actuator load autonomously, operating with no prior knowledge of the excitation applied, its only input being the physical excitation of the structure itself. To test the system, a shaker is attached to the structure and a stepped sinusoidal input is applied. Each frequency is held constant for ten seconds at intervals of $2 \mathrm{~Hz}$ and the vibration data from the accelerometers are logged to record a full time history. This test is carried out first with the control 
system activated, and then with it deactivated. The resulting time histories are shown in figure 14(a). A clearer picture of the results is obtained from the root mean square (RMS) plot in figure 14(b). The most striking feature is the marked reduction in response in the region of the $50 \mathrm{~Hz}$ mode. In fact, the true extent of the improvement is greater than can be seen, as the measurement from the accelerometer in the nonadaptive experiment was clipped at $1 \mathrm{~ms}^{-} 2$ by the ADC. The adaptive system successfully avoids the peak of the resonance in this region. This is an important result: it demonstrates that without any damping, and without the use of fully active control systems, the driving frequency can traverse a region containing a resonant mode of vibration without exciting that mode. This could be very useful, for example, in rotor spin-up. Furthermore, the force required from the actuator to acheive this goal is unrelated to the level of force generated by the excitation signal, so that a well-designed system based on this principle will use far less power than an active system directly countering the dynamic loads.

\section{IV.D. Areas for Development}

Despite the success of this preliminary study, many opportunities for improvement are immediately presented. These are broadly separated into three groups: frequency estimation, route planning, and overall implementation.

Shortcomings of the frequency estimation algorithm were recognised at its conception. It is quite basic and does not always correctly identify the driving frequency. There are two separate issues which must be addressed. The first of these will be referred to here as skipping. Skipping occurs when the structure is excited at a constant frequency but the algorithm temporarily loses track of this and identifies another frequency as the driving frequency. It results in the output alternating rapidly between values, and the actuator constantly trying to adjust to the appropriate load. Not only is this inefficient for power consumption but it can also result in higher levels of structural response. Two examples can be seen as a series of spikes in the response at around $35 \mathrm{~Hz}$ and $95 \mathrm{~Hz}$. The second issue with the frequency estimation is simply one of accuracy. The results from this algorithm were seen to wander above and below the known excitation values. This can cause a considerable lack of performance in situations where the natural frequencies are closely spaced or where the sensitivity of modes to the actuation is low. In these cases the exact excitation frequency must be known to avoid resonance. Better recognition systems need to be developped, perhaps incorporating several sensors and accounting for the specific response characteristics of the structure.

For this study the route planning was performed manually using the FRF results from just one structural location, and was based only on one parameter: the driving frequency. It is proposed in the first instance to develop route planning algorithms to automate this task. These should be based on a range of FRFs from around the structure, possibly weighted by their respective significance. Once this is achieved, the next goal to focus on should be finding the path that creates an acceptable balance between response reduction and power consumption by minimising the change in actuation required throughout the frequency spectrum. Further enhancements to the system could then be made through incorporating other parameters to be taken into consideration when deciding the optimum actuator load. These could include: actual structural response (if it is relatively low already, changes in actuation could be detrimental), the time derivative of the excitation frequency (attempting to predict where the actuator should be aiming for rather than where it should be now), where the actuator is now (for abrupt changes in frequency there may be a closer optimum position than if the frequency were to approach a given value gradually), and ultimately structural variability.

Structural variability is a difficult problem to overcome, but some aspects of it have obvious posibilities for exploitation. With sufficient sensors the state of the structure could be assessed and the appropriate loads applied accordingly. For example, simple strain gauges could provide further improvements in situations where external forces were known to routinely affect the strucutre, such as the tail rotor loads discussed in section II. Another proposition is that a learning algorithm could be employed so that the system could learn to adapt to gradual changes in the structural response characteristics over long time periods.

The overall implementation of this technique primarily concerns the placement of the actuator. The best location for influencing each mode can be assessed through sensitivity studies. Also, the effectiveness will be greatly enhanced by the inclusion of more than one actuator. Every mode will respond differently to any given actuation point, so by placing a network of actuators around the structure greater control is achieved. It is worth noting, however, that within a statically determinate structure the inclusion of more than one actuator will not provide any further benefits. 


\section{Conclusions}

The effect of structural loading on dynamic response has been demonstrated in a number of ways; some of these forces are undesirable contributors to measurement variability, whereas others have been deliberately applied to produce a desired result. However, all of the examples given in this paper show that significant alterations to the dynamic characteristics can be made through applied forces. Methods of modelling the behaviour have been explored and joints have been identified as a potential problem area for modelling purposes. It is found that model parameters updated for the zero load case will, in general, not apply across the full loading range, but that it may be possible to parameterize the change in the parameters themselves with load, avoiding the need to update each load case individually.

An automated response suppression system has been implemented, and this has been shown to successfully reduce the overall response of an arbitrary structure to narrow-band excitation across a range of frequencies. This is achieved without the use of damping or active control, but using an adaptive technique which requires less power than similar active solutions. This is only a preliminary study allowing the identification of immediate areas for improvement. These include better frequency estimation algorithms, more advanced selection of the optimum loading, and informed placement of one or more actuators. Tools such as sensitivity analysis have been proposed to help achieve these goals. The demonstration serves as a proof-of-concept and the successful results provide a good foundation for further work in this direction.

\section{Acknowledgments}

Thanks are due to Clive Rendall and Tony Griffith for their invaluable practical expertise and advice, without which this project would be progressing much more slowly.

\section{References}

${ }^{1}$ Warburton, G. B., The Dynamical Behaviour of Structures, Pergamon Press, 2nd ed., 1976.

${ }^{2}$ Stephens, B. C., "Natural Vibration Frequencies of Structural Members as an Indication of End Fixity and Magnitude of Stress," Journal of the Aeronautical Sciences, Vol. 4, 1936, pp. 54-56.

${ }^{3}$ J., O. and P., D. H. J., "Theory of the Dynamic Vibration Absorber," Transactions of the ASME, Journal of Applied Mechanics, Vol. 50, No. 7, 1928, pp. 9-22.

${ }^{4}$ T., P. J., M., G. R., and I., L., "Active Control of Helicopter Vibration," Computing and Control Engineering Journal, Vol. December, 1994, pp. 277-284.

${ }^{5}$ A., C. K., "State-Switched Absorber for Vibration Control of Point-Excited Beams," Journal of Intelligent Material Systems and Structures, Vol. 13, 2002, pp. 97-105.

${ }^{6}$ J., L. and K., L., "Tunable Electromagnetic Vibration Absorber: Characterisation and Application," Journal of Sound and Vibration, Vol. 295, 2006, pp. 708-724.

${ }^{7}$ Coote, J. E., "Consideration of Dynamic Variability," Tech. Rep. Internal Report, University of Bristol, Bristol BS8 1TR, England, August 2006.

${ }^{8}$ Crocombe, A. D., "How to Tackle Nonlinear Finite Element Analysis," NAFEMS, Glasgow, 2001.

${ }^{9}$ Cook, R. D., Concepts and Applications of Finite Element Analysis, John Wiley \& Sons, Inc., 1974.

${ }^{10}$ Mayo, J. M., García-Vallejo, D., and Domínguez, J., "Study of the Geometric Stiffening Effect: Comparison of Different Formulations," Multibody System Dynamics, Vol. 11, 2004, pp. 321-341.

${ }^{11}$ Przemieniecki, J. S., Theory of Matrix Structural Analysis, McGraw-Hill, 1968.

12 Jennings, A., "Frame Analysis Including Change of Geometry," Proceedings of the ASCE, Journal of the Structural Division, Vol. 94, 1968, pp. 627-644.

${ }^{13}$ Cook, R. D., Finite Element Modelling for Stress Analysis, John Wiley \& Sons, Inc., 1994.

${ }^{14}$ Igusa, T., "Critical Configurations of Systems Subjected to Wide-Band Input," Journal of Sound and Vibration, Vol. 168, No. 3, 1993, pp. 525-541.

${ }^{15} \mathrm{du}$ Bois, J. L., Adhikari, S., and Lieven, N. A. J., "Experimental and Numerical Investigation of Mode Veering in a Stressed Structure," Proceedings of the 25th International Modal Analysis Conference, 2007, pp. 233.

${ }^{16}$ I., F. M. and E., M. J., Finite Element Model Updating in Structural Dynamics, Kluwer Academic Publishers, 1995.

${ }^{17}$ Ewins, D. J., "Model validation: Correlation for Updating," Sādhanāa, Vol. 25, No. 3, 2000, pp. 221-234.

${ }^{18}$ J. E. Mottershead, C. Mares, M. I. F. and James, S., "Selection and Updating of Parameters for an Aluminium SpaceFrame Model," Mechanical Systems and Signal Processing, Vol. 14, No. 6, 2000, pp. 923-944.

${ }^{19}$ Starling, Electricity and Magnetism, Longmans, Green and Company, London, 1929.

${ }^{20}$ P., L. B., Signal Processing and Linear Systems, Berkeley-Cambridge Press, CA, USA, 1998. 


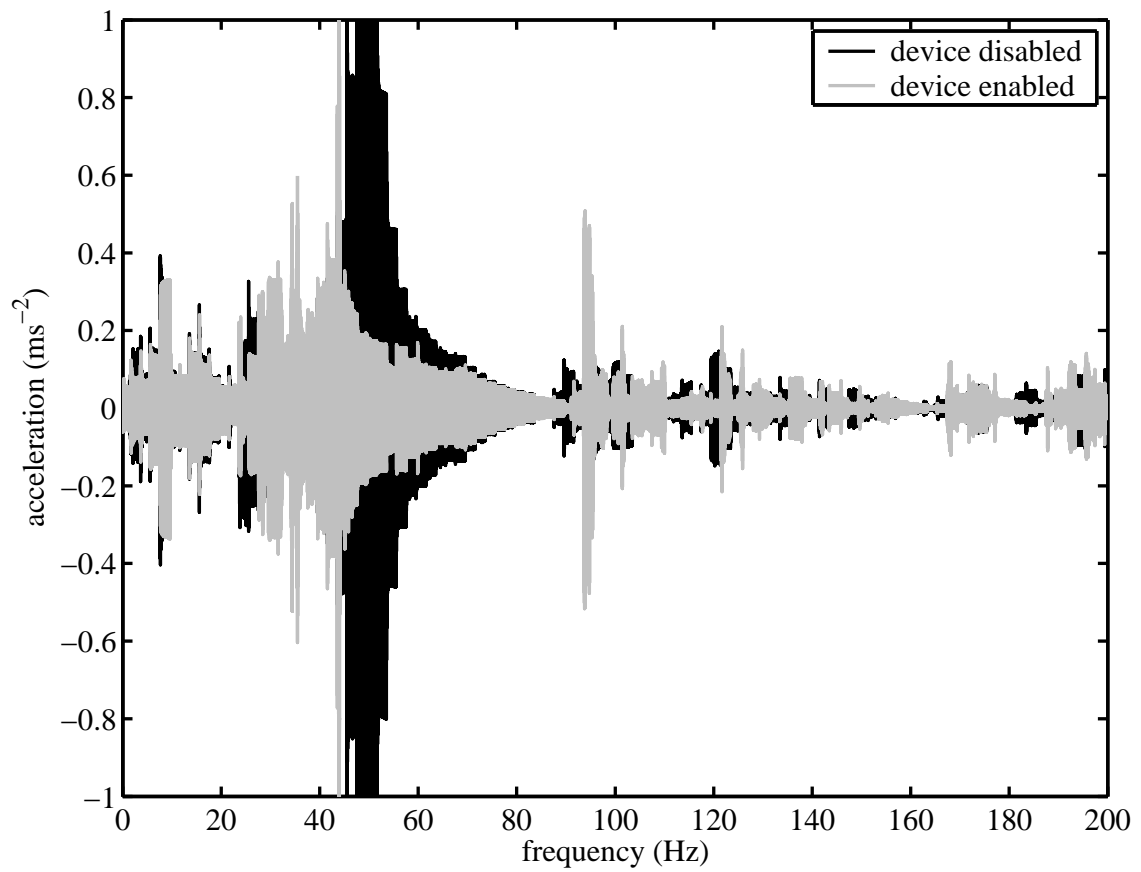

(a) response time history

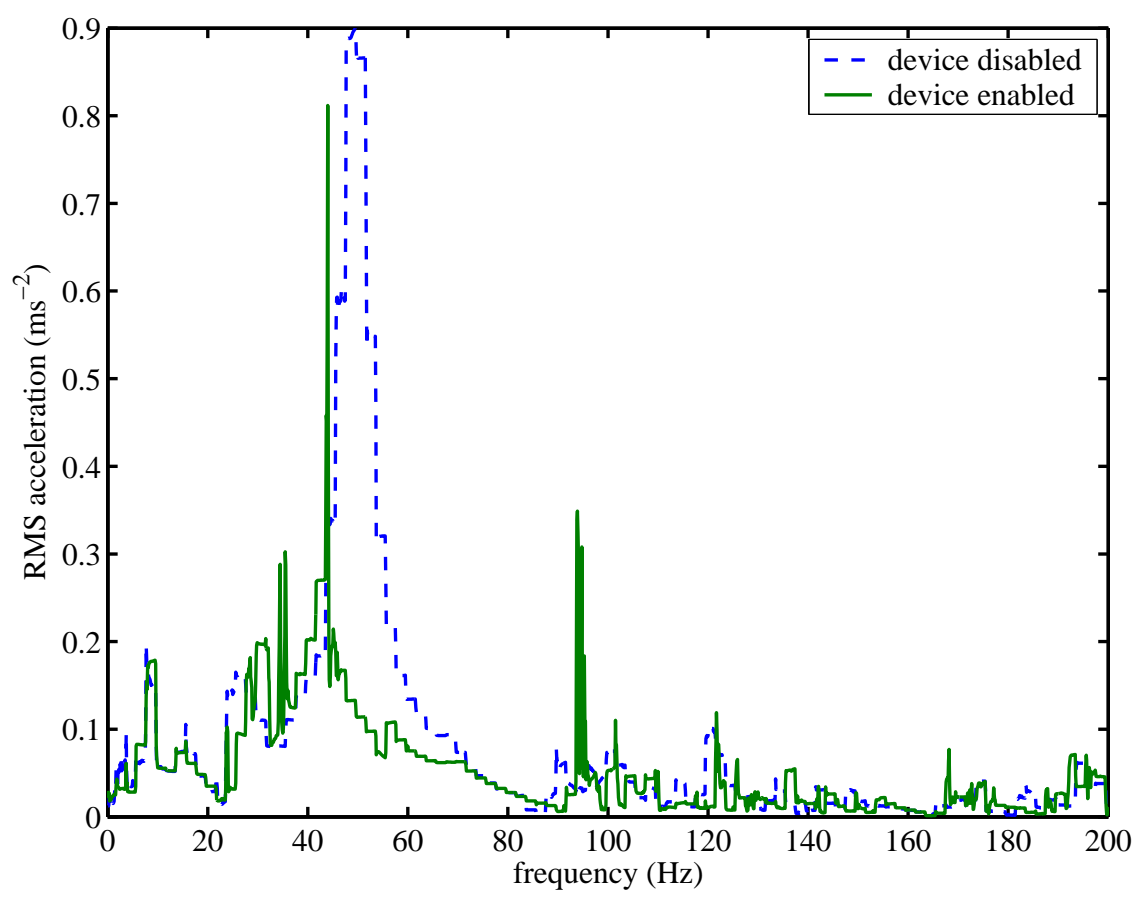

(b) RMS response time history

Figure 14. Time histories of the response of the structure on the cubic diagonal. The excitation signal is a stepped sin wave, incrementing once every 10 seconds. The horizontal axes on the graphs indicate the excitation frequency (found by dividing the time in seconds by 5 ). 